\title{
ChemSusChem
}

Chemistry-Sustainability-Energy-Materials

\section{Chemistry Europe}

European Chemical Societies Publishing

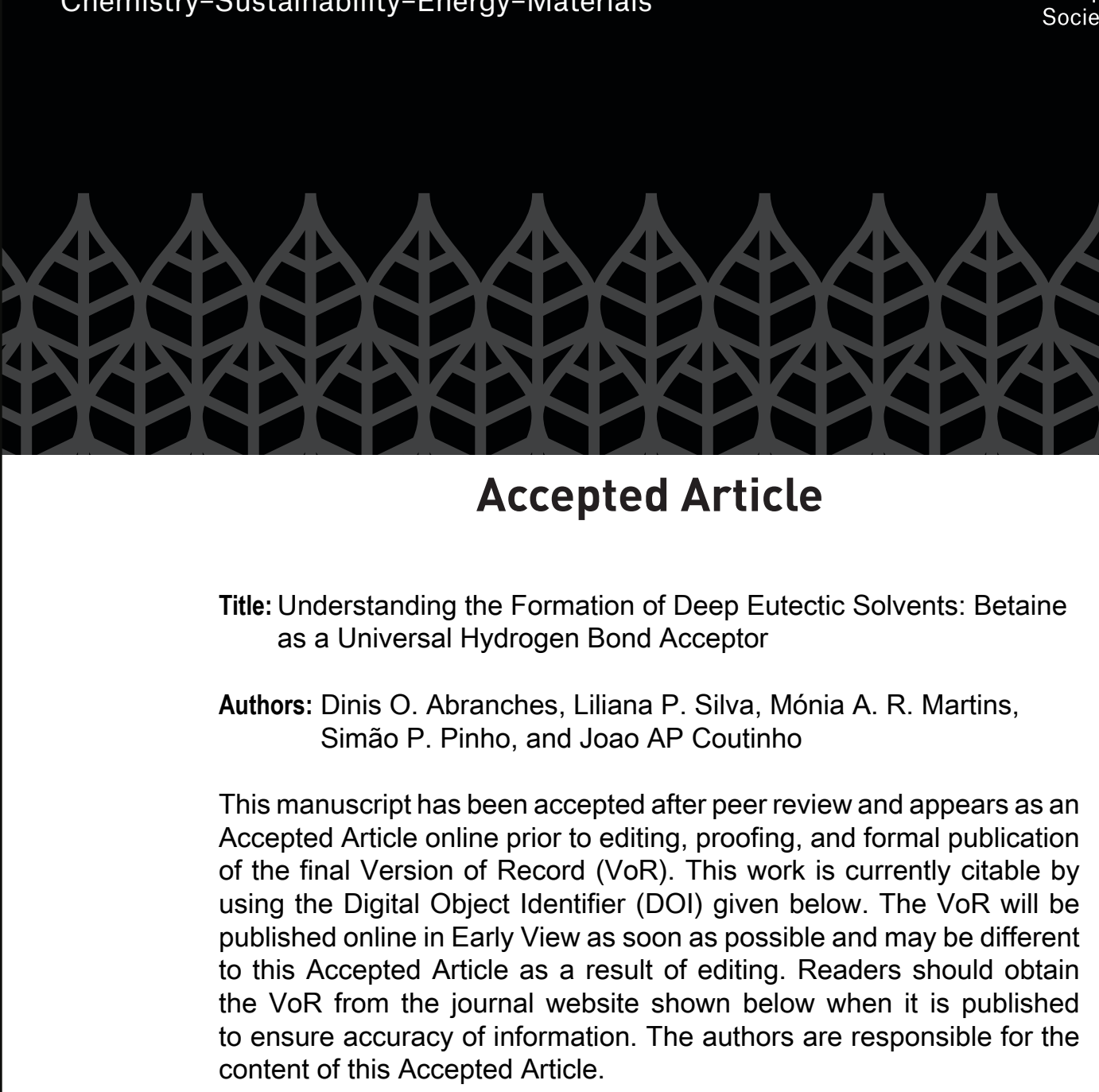

To be cited as: ChemSusChem 10.1002/cssc.202001331

Link to VoR: https://doi.org/10.1002/cssc.202001331 


\title{
Understanding the Formation of Deep Eutectic Solvents: Betaine
} as a Universal Hydrogen Bond Acceptor

\author{
Dinis O. Abranches, ${ }^{[a]}$ Liliana P. Silva, ${ }^{[a]}$ Mónia A. R. Martins, ${ }^{[a]}$ Simão P. Pinho ${ }^{[b]}$ and João A. P. \\ Coutinho*[a]
}

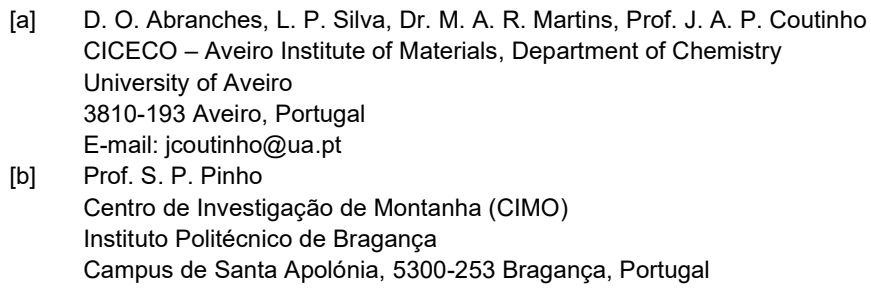

Supporting information for this article is given via a link at the end of the document.

\begin{abstract}
The mechanism of formation of betaine-based deep eutectic solvents (DES) is here presented for the first time. Due to its polarity unbalance, it is found that betaine displays strong negative deviations from ideality when mixed with a variety of different organic substances. These results pave the way for a comprehensive design of novel deep eutectic solvents. A connection to biologically relevant systems is made using betaine (osmolyte) and urea (protein denaturant), showing that these two compounds form a DES whose molecular interactions are greatly enhanced in the presence of water.
\end{abstract}

Deep eutectic solvents (DES) are liquid mixtures prepared by mixing two or more solid substances, whose components present significant negative deviations to thermodynamic ideality in the liquid state. ${ }^{[1,2]}$ Abbott and co-workers ${ }^{[3,4]}$ proposed, at the beginning of this century, this class of green solvents that and have been successfully used in separation processes, extractions, chemical reactions, and electrochemistry applications. ${ }^{[5,6]}$ Among other relevant features stands their ability to have their properties fine-tuned by the choice of their precursors.

The most commonly used compound to prepare DES is cholinium chloride (choline). ${ }^{[5,6]}$ Although it has been shown that this compound has an ideal behavior in most so-called DES reported in the literature, ${ }^{[1]}$ it remains an attractive DES-forming compound due to its low toxicity, high biodegradability, low cost, and widespread availability. ${ }^{[7]}$ Its ability to induce the necessary melting temperature depression for the formation of DES stems from its remarkably low enthalpy of fusion $(4.3 \mathrm{~kJ} / \mathrm{mol})$ rather than the formation of particularly strong intermolecular interactions. ${ }^{[1,8,9]}$

Trimethylglycine (henceforth betaine) has been used to prepare DES by some authors. Zeng et al. ${ }^{[10]}$ demonstrated the formation of DES by mixing betaine and urea. They were unable to detect the freezing point of several betaine/urea mixtures, observing, instead, glass transitions at a temperature of around $50{ }^{\circ} \mathrm{C}$. Cardellini et al., ${ }^{[11]}$ studied the formation of deep eutectic solvents by combining betaine with 23 different aromatic carboxylic acids and 13 aliphatic carboxylic acids. Other authors have reported the application potential of betaine-based deep eutectic solvents, ${ }^{[12-17]}$ and some studies on their properties are also available. ${ }^{[18-21]}$

Much like choline, betaine is non-toxic and readily biodegradable. However, unlike choline, betaine is industrially obtained from renewable (instead of fossil) resources as a byproduct of sugar production. Betaine is also an osmolyte and plays important roles in the cells of most living organisms. Despite its sustainable character, betaine-based DES are still poorly studied, and their intermolecular interactions not explored nor understood. Zeng et al. ${ }^{[10]}$ pointed out that typical hydrogen bonds could not account for the formation of the betaine/urea DES. This lack of fundamental knowledge is heightened by the scarcity of solid-liquid equilibrium data for betaine-based systems, which hampers the rational development of novel betaine-based DES leaving them to the chance of tedious experimental trial and error essays.

This work aims to lay the foundations for the rational design of novel deep eutectic solvents based on betaine. To do so, the solid-liquid phase diagrams of several betaine-based DES are herein measured to understand the molecular interactions of the components on these systems. Solid-liquid equilibrium data are a convenient way to infer about molecular interactions: a component of a mixture is said to have negative/positive deviations to ideality if the experimental melting temperature of the mixture is lower/higher than that predicted by assuming ideality (ideal liquidus line). In turn, negative/positive deviations to ideality reveal that the component is establishing stronger/weaker intermolecular interactions in the mixture than in its pure (hypothetical or not) liquid state.

Betaine is a zwitterion, i.e., it possesses both formal positive and negative charges. As such, betaine should establish strong ion-ion interactions with itself. Since the formation of DES is achieved through negative deviations to ideality and considering that these deviations arise due to the formation of stronger interactions in the mixture than in the pure phases, the use of a zwitterion to prepare DES is, at first glance, inadequate. After all, ion-ion interactions are much stronger than the hydrogen bonding claimed to be responsible for the formation of DES. However, the formal positive charge of betaine is shielded by methyl groups (see Figures 1 and $\mathrm{S} 1$ for its polarity surface and chemical structure), which may inhibit electrostatic interactions with itself. As such, this compound possesses a polarity unbalance: it is an excellent hydrogen bond acceptor but a poor positive charge donor. Note here the double meaning of the expression excellent hydrogen bond acceptor: the carboxylate group of betaine is a strong hydrogen bond acceptor by itself, but the fact that its positive charge is highly shielded limits the extent of ion-ion 
interactions between betaine molecules, which facilitates negative deviations to ideality when mixed with other compounds.
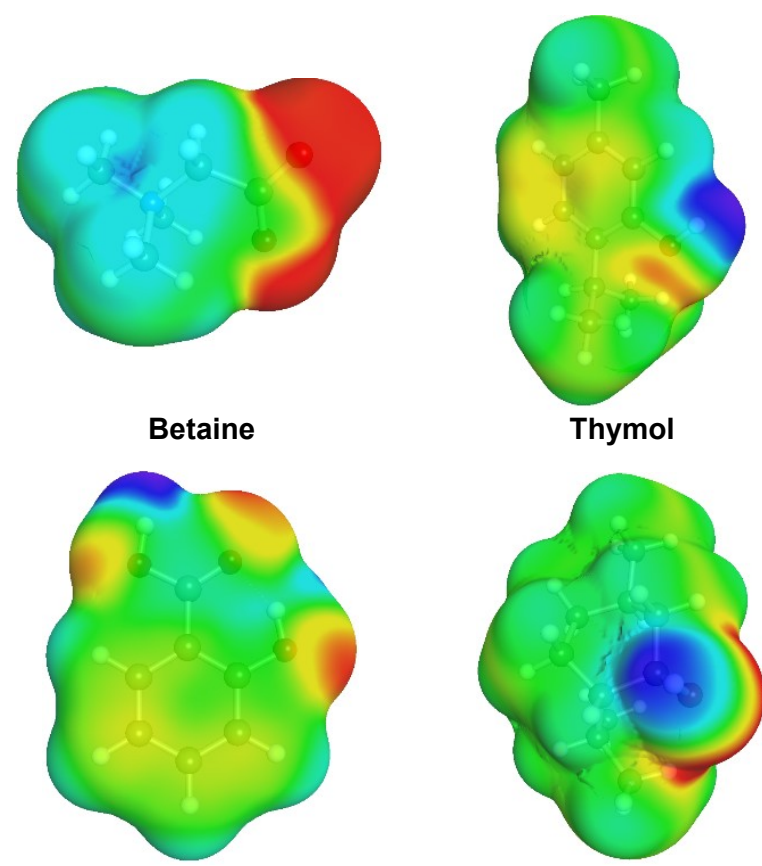

Salicylic Acid

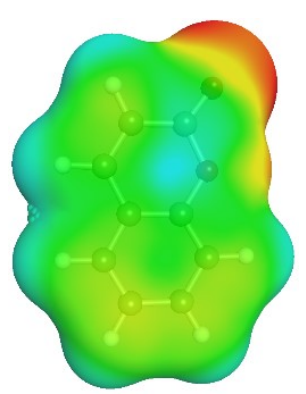

Coumarin

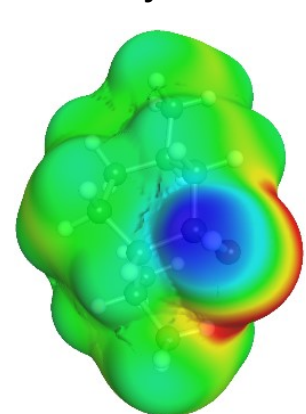

Menthol

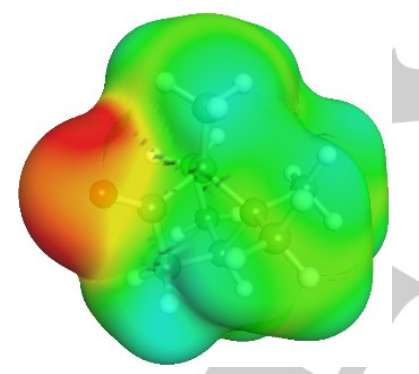

Camphor

Figure 1. Polarity surface ( $\sigma$-surface) of betaine and of the substances used in this work to probe the hydrogen bond acceptor character of betaine, calculated as explained in the experimental section.

The polarity unbalance of betaine bears a strong resemblance to Type V DES. ${ }^{[22]}$ Thymol, for instance, possesses a hydroxyl group directly attached to an aromatic ring. Due to resonance effects, its oxygen is less negative than usual, in opposition to its proton, which is more positive than usual. This has been explored to successfully prepare non-ionic DES whose components present strong negative deviations to ideality due to stronger hydrogen bonding in the mixture than in the pure liquid phases. ${ }^{[22]}$ Thus, considering their success as DES precursors, phenolic hydrogen bond donors are the starting point of the present work (see Figures 1 and S1 for its polarity surface and chemical structure).

To probe the hydrogen bond acceptor character of betaine, its binary mixture with two phenolic strong hydrogen bond donors (thymol or salicylic acid) are herein first studied. The solid-liquid phase diagrams of these systems, measured in this work, are reported in Figure 2, along with the ideal liquidus lines (as explained in the experimental section).

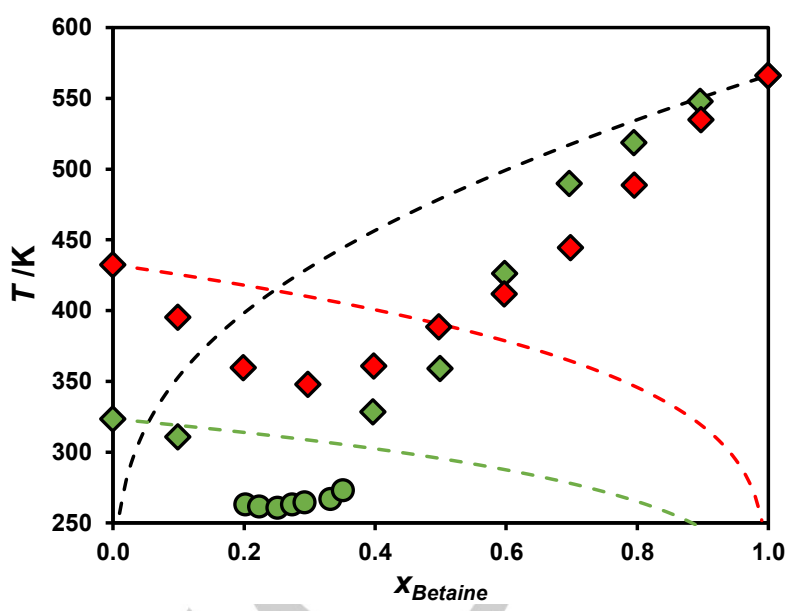

Figure 2. Experimental solid-liquid phase diagrams of the binary systems composed of betaine and thymol ( $\checkmark$ this work; 1 literature ${ }^{[15]}$ ) or salicylic acid $(\diamond)$, along with the ideal liquidus line of betaine $(---)$, thymol $(-=-)$ and salicylic acid $(=--)$.

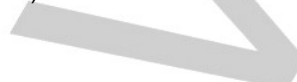

Figure 2 shows that betaine presents large negative deviations to ideality when mixed with thymol or with salicylic acid, implying that betaine has stronger interactions with these phenolic compounds than with itself. These results confirm the hypothesis that pure betaine cannot establish strong ion-ion interactions in its (hypothetical) liquid phase, due to the shielding of its positive charge. Hence, betaine has an energetic advantage to mix with thymol or with salicylic acid since the interaction established between the acidic proton of thymol or salicylic acid, and the carboxylate group of betaine is stronger than any interaction betaine is able to establish with itself. Figure 2 shows that thymol and salicylic acid also present significant negative deviations to ideality when mixed with betaine, arising from the strong hydrogen bonding between their acidic protons and the negatively charged carboxylate group of betaine.

Since betaine is an excellent hydrogen bond acceptor, unable to establish strong interactions with itself, the magnitude of its negative deviations to ideality in a mixture should correlate with the hydrogen donning ability of the second component. To explore this idea, the solid-liquid phase diagrams of binary mixtures composed of betaine and menthol, coumarin, or camphor were measured in this work and are reported in Figure 3. Note that menthol is both a regular hydrogen bond donor and acceptor, while coumarin is a regular hydrogen bond acceptor but a weak hydrogen bond donor and camphor possesses no hydrogen bond donning capability whatsoever This concept is further developed and quantified in section S2.5 of the Supporting Information, where the negative deviations to ideality of betaine are successfully correlated against computationally developed polarity factors. 


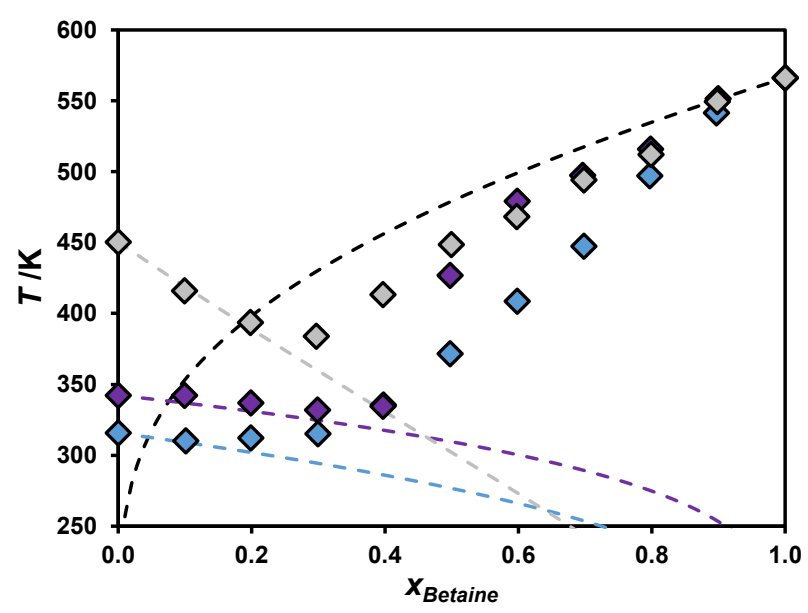

Figure 3. Experimental solid-liquid phase diagrams of the binary systems composed of betaine and menthol $(\diamond)$, coumarin $(\diamond)$ or camphor $(\diamond)$, along with the ideal liquidus lines of betaine $(---)$, menthol $(=--)$, coumarin $(-=-)$ and camphor $(=--)$.

Figure 3 shows that betaine presents significant negative deviations to ideality when mixed with menthol, yet weaker than those observed for the betaine/thymol and betaine/salicylic acid systems. Furthermore, betaine also displays negative deviations to ideality when mixed with coumarin, which is only a weak hydrogen bond donor, supporting the hypothesis that betaine is an excellent hydrogen bond acceptor with negative deviations to ideality even when mixed with weak hydrogen bond donors. Surprisingly, camphor is also able to induce mild negative deviations to ideality in betaine. This is rather intriguing since camphor possesses no hydrogen bond donning capability. Albeit there is some uncertainty concerning the enthalpy of fusion of betaine (see discussion in section S2.7 of Supporting Information), its hypothetical pure liquid phase must be very organized to minimize negative charge repulsion, with a low entropy (as also suggested by its low value of entropy of fusion listed in Table S2). Therefore, it displays negative deviations to ideality when mixed with large apolar molecules, because it allows the increase of the entropy of the liquid phase by shielding the betaine negative charges.

Having discussed the molecular interactions responsible for the formation of betaine-based DES, its thermodynamic behavior is now compared to cholinium chloride. Such comparison is useful since i) choline is the prototypical hydrogen bond acceptor (HBA) used to prepare DES and ii) betaine and choline are similar from a structural point of view, since both possess a formal positive charge shielded by alkyl groups (see Figure $\$ 2$ for chemical structure comparison). Therefore, the solid-liquid phase diagrams of the binary systems composed of choline and the same compounds shown in Figures 1 and 2 were measured in this work and reported in Figure S3. Unlike betaine, choline behaves ideally when mixed with all the compounds studied. This is not an indication, though, that strong ion-dipole interactions are not being formed between the chloride anion of choline and the positive protons of the other components. It is evident that thymol, for example, can act as a hydrogen bond donor and establish a strong hydrogen bond with the chloride anion. The major difference between betaine and choline, however, is that the later establishes strong hydrogen bonding with itself (between the hydroxyl group of the cholinium cation and the chloride anion), whereas the former cannot establish strong interactions between its carboxylate group and the shielded quaternary nitrogen.

The stark contrast between the behavior of betaine and choline reinforces the notion that the presence of hydrogen bonding in a system is neither a necessary nor sufficient condition for its components to display negative deviations to ideality, nor does it mean that the system is a deep eutectic solvent. Furthermore, from an application perspective, the eutectic temperatures of the betaine-based and choline-based systems here studied are similar (see Figure S4 for a system by system comparison). Thus, and considering the greener character of betaine (non-toxic, biodegradable, renewable, cheap, osmolyte in biological systems, widespread industrial use), its potential and relevance as a DES-forming compound becomes apparent.

The asymmetric thermodynamic behavior of some of the betaine-based DES reported in Figure 3 is akin to that observed in tetramethylammonium chloride-based systems. ${ }^{[23]}$ There is a clear analogy between tetramethylammonium chloride $\left(\left[\mathrm{N}_{1,1,1,1}\right] \mathrm{Cl}\right)$ and betaine: both compounds possess an easily accessible formal negative charge, and an alkyl group-shielded positive formal charge, as illustrated in Figure S2. To emphasize the similarity of these two compounds, the solid-liquid phase diagrams of the systems composed of betaine and octadecanoic acid, or octadecan-1-ol (octadecanol), were measured in this work. These results are reported in Figure S5, showing that betaine and $\left[\mathrm{N}_{1,1,1,1}\right] \mathrm{Cl}$ behave in the same manner, displaying strong negative deviations to ideality, while octadecanol and octadecanoic acid behave ideally.

So far, the behavior of betaine in deep eutectic solvents has been reported, analyzed, and explained in terms of the molecular interactions with itself and the second component of the system. Yet a third component, water, is often present in DES due to their hygroscopic nature. Thus, studying the formation of DES in the presence of small amounts of water is essential and allows a connection to biologically relevant systems. Betaine is an excellent osmolyte and, curiously, can counteract the denaturing effect of urea on proteins. Although the mechanism behind this phenomenon is still under debate, studies have shown that this occurs due to strong interactions established between betaine, urea, and water in the bulk phase. Saladino et al. ${ }^{[24]}$ showed that betaine protected the denaturation of a peptide in presence of urea, not by directly interacting with the surface of the peptide but, instead, through some indirect effect on the water-urea bulk phase. This suggests a strong interaction between betaine and urea, which survives its dilution in water. Building on this concept, Kumar and Kishore ${ }^{[25]}$ reported a synergistic effect between betaine, urea and water, where the extent of hydrogen bonding between betaine and water increases in the presence of urea, while the extent of hydrogen bonding between water and urea also increases in the presence of betaine. Put differently, waterbetaine contacts increase in the presence of urea and water-urea contacts increase in the presence of betaine.

Taking the considerations above into account, the solidliquid phase diagrams of betaine/urea were measured in this work, without water and with $2 \mathrm{wt} \%$ of water, and are reported in Figure 4. The results reveal significant phenomena. First, unlike choline, betaine presents a severe negative deviation from ideality. Second, urea also displays negative deviations to ideality, revealing stronger interactions between urea and betaine than those observed in their pure phases. Third, Figure 4 shows that the addition of a small amount of water greatly increases the 
deviations to ideality presented by both betaine and urea. Adding $2 \mathrm{wt} \%$ of water lowers the eutectic temperature from $80^{\circ} \mathrm{C}$ to a temperature lower than $20^{\circ} \mathrm{C}$. In fact, the mixtures with a betaine mole fraction of 0.3 or 0.4 did not recrystallize even when kept at a temperature of $-80^{\circ} \mathrm{C}$ for $72 \mathrm{~h}$. This is consistent with the results of Zeng et al. ${ }^{[10]}$, where it was reported that betaine/urea mixtures (prepared without drying the compounds) did not display crystallization, showing, instead, glass transitions at a temperature of around $-50^{\circ} \mathrm{C}$.

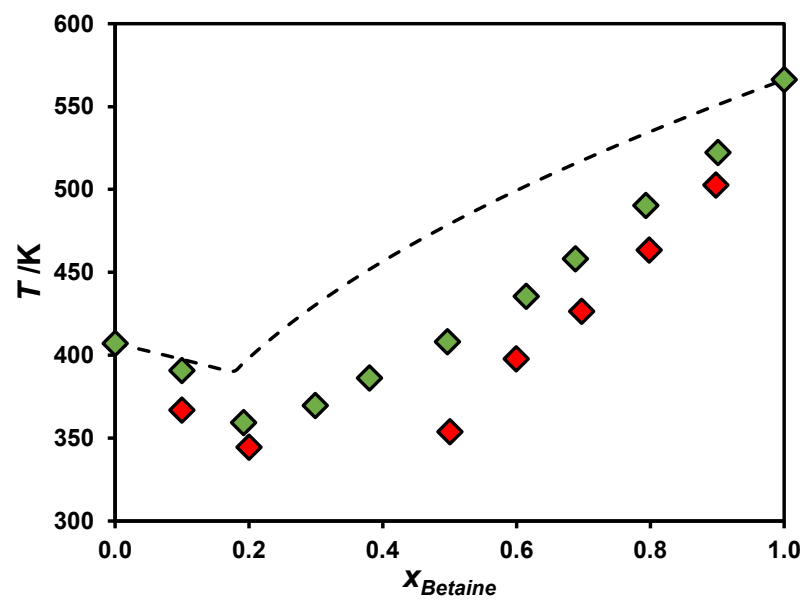

Figure 4. Experimental solid-liquid phase diagrams of the binary system composed of betaine and urea without added water $(\diamond)$ and with 2 wt\% water $(\diamond)$, along with the ideal solid-liquid equilibrium phase diagram (- - -).

The effect of water on betaine/urea may result from its small size and high hydrogen bonding ability that may stabilize betaine in the liquid phase by shielding its electrostatic repulsions and mediating its interaction with urea. At this point, the nature of this specific interaction is unknown, and further research is needed. However, it is interesting to note that there is a sharp difference between the crystal structure of anhydrous betaine and the crystal structure of monohydrated betaine. In the former, ${ }^{[26]}$ the carboxylate moiety interacts with the quaternary ammonium moiety, as expected; however, in the latter, ${ }^{[27]}$ a bridge between two carboxylate groups is created by two water molecules. This, coupled with the counterintuitive synergistic behavior reported by Kumar and Kishore ${ }^{[25]}$ where water increases its hydrogen bonding with both betaine and urea at the same time, suggests some sort of water molecule bridge between betaine and urea, akin to that observed in the crystal structure of monohydrated betaine. Using DFT (see section S2.6 of Supporting Information), a remarkably strong betaine/urea/water complex of this nature was identified, in which water bridges the carboxylate group of betaine with a proton of the amine group of urea. This complex is more energetically favorable than any interaction established in the betaine/urea system and, thus, may be the cause for the strong negative deviations of this system.

The impact of water in the betaine/urea system is unprecedent and huge compared to the effect of water on other deep eutectic solvents, such as the choline/urea system. ${ }^{[28]}$ The impact of adding $2 \mathrm{wt} \%$ of water to the systems betaine/menthol and betaine/sorbitol was also probed in this work by measuring the solid-liquid phase diagrams of these systems (see Figure S6). Again, the effect of water on their melting temperature is much smaller than that observed for betaine/urea mixtures. Understanding the molecular interactions responsible for the striking behavior of water in the betaine/urea system has significant implications for the field of DES and NADES (natural deep eutectic solvents), since not only does Figure 4 report a DES with high application potential, whose eutectic temperature can be tailored by the addition of a small amount of water, it also supports the mechanisms proposed for the biological role of betaine, strongly encouraging the paradigm of NADES being the missing link in understanding cellular physiology. ${ }^{[29]}$

In conclusion, it was here shown that betaine possesses a charge unbalance making it an excellent hydrogen bond acceptor since it cannot establish strong ion-ion interactions with itself. Consequently, betaine will form deep eutectic solvents with organic substances that possess hydrogen bond donning capability. The negative deviations to ideality presented by betaine in these mixtures correlate with the hydrogen bond donning strength of the other component, with betaine showing negative deviations even for weak hydrogen bond donors. Thus, betaine can be seen as a non-selective, universal DES-forming hydrogen bond acceptor. This, coupled with its renewable character, non-toxicity, biodegradability, low cost, and widespread use, asserts betaine as a powerful choice to prepare novel deep eutectic solvents. Finally, the molecular mechanisms discussed here are suggested as key to understand the osmolyte potential of betaine, such as in the biologically relevant betaine/urea system, whose eutectic temperature is strongly affected by the presence of a small amount of water.

\section{Experimental Section}

\section{Materials}

The substances trimethylglycine (betaine), cholinium chloride (choline), thymol, salicylic acid, (-)-menthol (menthol), coumarin, (1R)-(+)-camphor (camphor), octadecan-1-ol (octadecanol), octadecanoic acid, urea and sorbitol were experimentally used in this work. Their CAS number, supplier, purity and water content are listed in Table S1. Due to their hygroscopicity, betaine and choline were dried before use by stirring under vacuum $(0.1 \mathrm{~Pa})$ at room temperature $(298 \mathrm{~K})$ for at least $72 \mathrm{~h}$. The water content of all compounds was measured using a Metrohm 831 Karl Fischer coulometer, with the analyte Hydranal Coulomat AG from Riedel-de-Haen.

\section{Solid-Liquid Phase Diagrams}

The solid-liquid phase diagrams of the binary systems composed of betaine and thymol, salicylic acid, menthol, coumarin, camphor, octadecanol, octadecanoic acid, urea or sorbitol, and composed of choline and thymol, salicylic acid, menthol, coumarin or camphor were experimentally measured in this work. To do so, the appropriate amount of each component was weighted using an analytical balance (model ALS 220-4N from Kern) with a readability of $0.1 \mathrm{mg}$, and mixtures were prepared to cover the entire concentration range of each system. Then, each mixture was heated under stirring until fusion and allowed to recrystallize, in order to ensure intimate contact between both components in the solid phase. Once recrystallized, the mixtures were crushed with a mortar and pestle and the 
resulting powder was used to fill glass capillaries. Due to the hygroscopicity of both betaine and choline, this procedure was done inside a dry-argon glovebox to avoid water contamination. Finally, the melting temperature of each sample was measured using a melting point device model M-565 from Büchi (temperature resolution of $0.1 \mathrm{~K}$ ) and a temperature gradient of $0.1 \mathrm{~K} / \mathrm{min}$. The melting temperature was taken as the temperature at which complete fusion is observed and was measured thrice for each mixture.

The procedure described in the last paragraph was also used to prepare the systems with $2 \mathrm{wt} \%$ of water. The appropriate amount of water was added whilst preparing the mixtures. The water content of the betaine-based mixtures with $2 \mathrm{wt} \%$ of added water was rechecked using the aforementioned Metrohm $831 \mathrm{Karl}$ Fischer coulometer and confirmed to be $2 \mathrm{wt} \%$.

In the cases of the system betaine/sorbitol/water, and the betaine/thymol mixture with a betaine mole fraction of 0.1 , the recrystallized samples possessed a paste-like consistency, which impeded the correct loading of the capillary glasses. In these instances, the melting temperature of the samples was measured in an oil bath, instead of the melting point device, using a Pt100 probe with $\pm 0.1 \mathrm{~K}$ precision.

\section{Solid-Liquid Equilibrium}

The solid-liquid equilibrium of a given component in a system of the eutectic type, provided it solidifies from the liquid mixture as a pure solid, is described by the following expression: ${ }^{[30,31]}$

$\ln \left(x_{i} \cdot \gamma_{i}\right)=\frac{\Delta_{m} h_{i}}{R} \cdot\left(\frac{1}{T_{m, i}}-\frac{1}{T}\right)$

where $x_{i}$ is the mole fraction of component $i, \gamma_{i}$ is its activity coefficient in the liquid phase, $T_{m, i}$ is its melting temperature, $\Delta_{m} h_{i}$ is its enthalpy of fusion (at temperature $T_{m}$ ), $R$ is the ideal gas constant and $T$ is the absolute temperature of the system. If experimental solid-liquid equilibrium data is available for a given binary system, the activity coefficients of its components can be easily calculated by rearranging Equation 1 :

$\gamma_{i}=\frac{\exp \left[\frac{\Delta_{m} h_{i}}{R} \cdot\left(\frac{1}{T_{m, i}}-\frac{1}{T}\right)\right]}{x_{i}}$

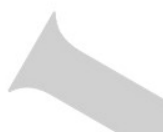

Whenever a component displays solid-solid transitions in its pure solid phase at a given temperature $T_{t, i}$, its solid-liquid equilibrium is still described by Equation 1 when $T>T_{t, i}$ but a second term needs to be added to the Equation when $T<T_{t, i}$ :

$\ln \left(x_{i} \cdot \gamma_{i}\right)=\frac{\Delta_{m} h_{i}}{R} \cdot\left(\frac{1}{T_{m, i}}-\frac{1}{T}\right)+\frac{\Delta_{t} h_{i}}{R} \cdot\left(\frac{1}{T_{t, i}}-\frac{1}{T}\right)$

where $\Delta_{t} h_{i}$ is the enthalpy of the solid-solid transition (at temperature $T_{t, i}$ ).

Throughout this work the ideal liquidus lines of the components of the systems studied and their activity coefficients are calculated using Equations 1 and 2, respectively, and the melting properties listed in Table S2. The single exception was that of the system thymol/cholinium chloride, where Equation 3 was used to calculate the ideal liquidus line of cholinium chloride due to its solid-solid transition ${ }^{[32]} \quad\left(T_{t}=352.9 \mathrm{~K}\right.$ and $\Delta_{t} h=17.2 \mathrm{~kJ} / \mathrm{mol}$ ).

The entropy of fusion, which is not needed in Equations 1-3 but was used in the main text to justify the anomalous behavior of betaine, is calculated using:

$\Delta_{m} S=\frac{\Delta_{m} h_{i}}{T_{m, i}}$

\section{DFT Simulations}

DFT with the COSMO solvation model was used in this work to i) assign density charges to the three-dimensional surface ( $\sigma$ surface) of the molecules used in this work to prepare betainebased DES and ii) optimize and calculate the interaction energy of the most stable molecular pairs in the betaine/urea/water system. All DFT calculations were performed using the COSMOBP-TZVP template of the software package TmoleX ${ }^{[33]}$ (TURBOMOLE), which uses a TZVP basis set for all atoms, a DFT with the BP86 functional, and the COSMO solvation model (infinite permittivity).

The chemical structure and the $\sigma$-surface of each molecule used in this work to prepare betaine-based systems are depicted in Figure S1 (section S2.1). The interaction energy of the molecular pairs $\left(E_{\text {int }}\right)$ discussed in section $\mathbf{S} 2.6$ is defined as:

$E_{\text {int }}=E_{\text {pair }}-E_{1}-E_{2}$

where $E_{\text {pair }}, E_{1}$ and $E_{2}$ are the total energy (with outlying charge correction) of the molecule pair, and the isolated molecules, respectively. The interaction energy of the betaine-urea-water complex is defined as:

$E_{\text {int }}=E_{\text {complex }}-E_{\text {betaine }}-E_{\text {urea }}-E_{\text {water }}$

where $E_{\text {complex }}, E_{\text {betaine }}, E_{\text {urea }}$ and $E_{\text {water }}$ are the total energy (with outlying charge correction) of the betaine-urea-water complex, of betaine, of urea, and of water, respectively.

\section{Acknowledgements}

This work was developed within the scope of the projects CICECO-Aveiro Institute of Materials, UIDB/50011/2020 \& UIDP/50011/2020, financed by national funds through the Portuguese Foundation for Science and Technology/MCTES, and CIMO-Mountain Research Center, UIDB/00690/2020, financed by national funds through the FCT/MEC and when appropriate cofinanced by FEDER under the PT2020 Partnership Agreement.

Keywords: Trimethylglycine $\cdot$ Non-ideality $・$ Natural Deep Eutectic Solvents $•$ Green Chemistry $\bullet$ Zwitterions

M. A. R. Martins, S. P. Pinho, J. A. P. Coutinho, J. Solution Chem 2019, 48, 962-982

[2] L. J. B. M. Kollau, M. Vis, A. van den Bruinhorst, A. C. C. Esteves, R. Tuinier, Chem. Commun. 2018, 54, 13351-13354.

[3] A. P. Abbott, D. Boothby, G. Capper, D. L. Davies, R. K. Rasheed, J. Am. Chem. Soc. 2004, 126, 9142-9147. 
A. P. Abbott, G. Capper, D. L. Davies, R. K. Rasheed, V.

Tambyrajah, Chem. Commun. 2003, 70-71.

[5] Q. Zhang, K. De Oliveira Vigier, S. Royer, F. Jérôme, Chem. Soc. Rev. 2012, 41, 7108-7146.

[6] E. L. Smith, A. P. Abbott, K. S. Ryder, Chem. Rev. 2014, 114 11060-11082.

[7] K. Radošević, M. Cvjetko Bubalo, V. Gaurina Srček, D. Grgas, T. Landeka Dragičević, I. Radojčić Redovniković, Ecotoxicol. Environ. Saf. 2015, 112, 46-53.

[8] P. Pyykkö, ChemPhysChem 2019, 20, 123-127.

[9] L. Fernandez, L. P. Silva, M. A. R. Martins, O. Ferreira, J. Ortega, S. P. Pinho, J. A. P. Coutinho, Fluid Phase Equilib. 2017, 448, 9-14.

[10] C.-X. Zeng, S.-J. Qi, R.-P. Xin, B. Yang, Y.-H. Wang, J. Mol. Liq. 2016, 219, 74-78.

[11] F. Cardellini, M. Tiecco, R. Germani, G. Cardinali, L. Corte, L. Roscini, N. Spreti, RSC Adv. 2014, 4, 55990-56002.

[12] Y. Dai, J. van Spronsen, G.-J. Witkamp, R. Verpoorte, Y. H. Choi, Anal. Chim. Acta 2013, 766, 61-68.

[13] K. M. Jeong, J. Ko, J. Zhao, Y. Jin, D. E. Yoo, S. Y. Han, J. Lee, J. Clean. Prod. 2017, 151, 87-95.

[14] M. H. Zainal-Abidin, M. Hayyan, A. Hayyan, N. S. Jayakumar, Anal. Chim. Acta 2017, 979, 1-23.

[15] M. Tiecco, F. Cappellini, F. Nicoletti, T. Del Giacco, R. Germani, P. Di Profio, J. Mol. Liq. 2019, 281, 423-430.

[16] M. Espino, M. de los Ángeles Fernández, F. J. V. Gomez, M. F. Silva, TrAC Trends Anal. Chem. 2016, 76, 126-136.

[17] L. Duan, L.-L. Dou, L. Guo, P. Li, E.-H. Liu, ACS Sustain. Chem. Eng. 2016, 4, 2405-2411.

[18] I. M. Aroso, A. Paiva, R. L. Reis, A. R. C. Duarte, J. Mol. Liq. 2017, 241, 654-661.

[19] H. Kauppi, MSc thesis, Turku University of Applied Sciences (FI), 2019.

[20] Y. Elhamarnah, H. Qiblawey, M. S. Nasser, A. Benamor, Sci. Total Environ. 2020, 708, 134848.

[21] M. Rumyantsev, S. Rumyantsev, I. Y. Kalagaev, J. Phys. Chem. B 2018, 122, 5951-5960.

[22] D. O. Abranches, M. A. R. Martins, L. P. Silva, N. Schaeffer, S. P. Pinho, J. A. P. Coutinho, Chem. Commun. 2019, 55, 10253-10256.

[23] D. O. Abranches, R. O. Martins, L. P. Silva, M. A. R. Martins, S. P. Pinho, J. A. P. Coutinho, J. Phys. Chem. B 2020, acs.jpcb.0c02386.

[24] G. Saladino, S. Pieraccini, S. Rendine, T. Recca, P. Francescato, G. Speranza, M. Sironi, J. Am. Chem. Soc. 2011, 133, 2897-2903.

[25] N. Kumar, N. Kishore, J. Chem. Phys. 2013, 139, 115104.

[26] M. Viertorinne, J. Valkonen, I. Pitkänen, M. Mathlouthi, J. Nurmi, J. Mol. Struct. 1999, 477, 23-29.

[27] K. M. Harmon, G. F. Avci, J. Mol. Struct. 1984, 117, 295-302.

[28] X. Meng, K. Ballerat-Busserolles, P. Husson, J.-M. Andanson, New J. Chem. 2016, 40, 4492-4499.

[29] Y. H. Choi, J. van Spronsen, Y. Dai, M. Verberne, F. Hollmann, I. W. C. E. Arends, G.-J. Witkamp, R. Verpoorte, Plant Physiol. 2011, 156, 1701-1705.

[30] J. M. Prausnitz, R. N. Lichtenthaler, E. G. de Azevedo, Molecular Thermodynamics of Fluid-Phase Equilibria, Prentice Hall, Upper Saddle River, NJ, 1999.

[31] J. R. Elliott, C. T. Lira, Introductory Chemical Engineering Thermodynamics, Prentice Hall, Upper Saddle River, NJ, 1999.

\section{Coutinho, 2020.}

[33] TURBOMOLE V7.1 2016, a development of University of Karlsruhe and Forschungszentrum Karlsruhe $\mathrm{GmbH}, 1989-2007$, TURBOMOLE GmbH, since 2007; available from http://www.turbomole.com.

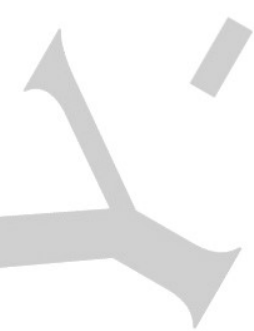

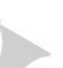

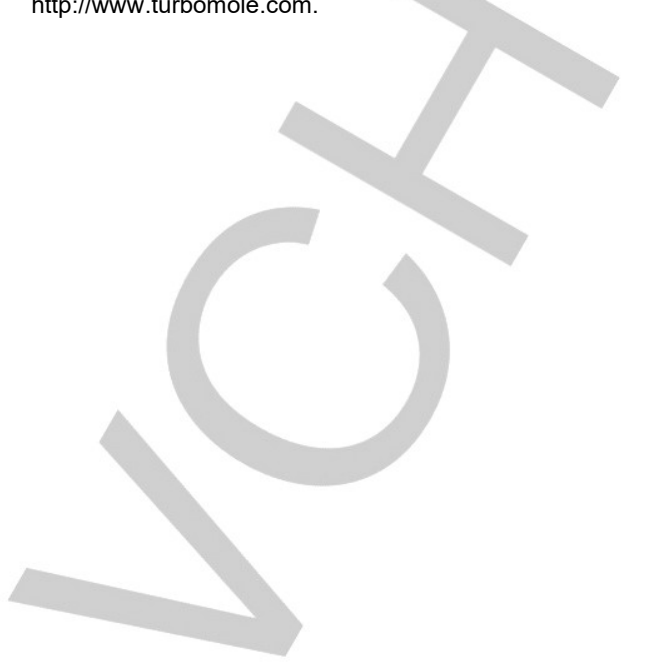




\section{Entry for the Table of Contents}
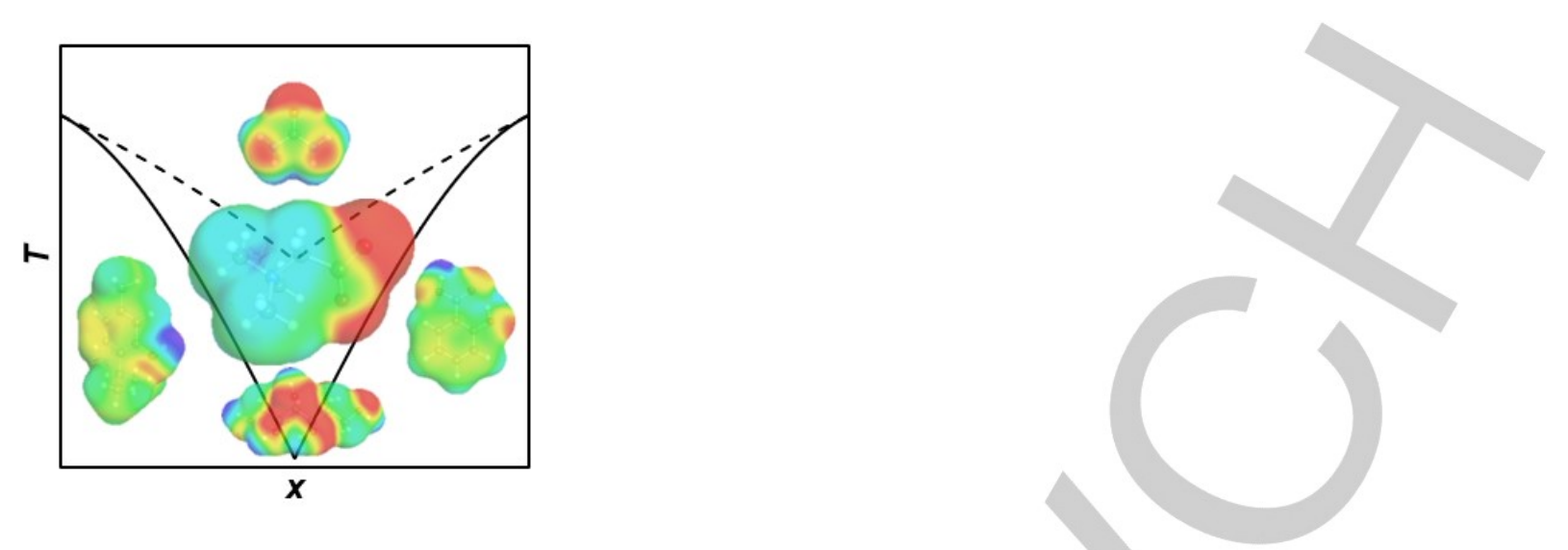

Betaine is an attractive DES-forming compound due to its sustainable character (non-toxic, biodegradable, renewable, cheap, osmolyte in biological systems, widespread industrial use). In this work the mechanism of formation of betaine-based deep eutectic solvents is presented and discussed in terms of molecular interactions. A particular system (betaine/urea) is found to be highly water sensitive, encouraging the paradigm of NADES being the missing link in understanding cellular physiology.

Institute and/or researcher Twitter usernames: @PATh_RG 